\title{
High brightness inductively coupled plasma source for high current focused ion beam applications
}

\author{
N. S. Smith, ${ }^{\text {a) }}$ W. P. Skoczylas, S. M. Kellogg, D. E. Kinion, and P. P. Tesch \\ FEI Company, 5350 NE Dawson Creek Drive, Hillsboro, Oregon 97124
}

O. Sutherland, A. Aanesland, and R. W. Boswell

Plasma Research Laboratory, Research School of Physical Sciences and Engineering,

The Australian National University, ACT 0200, Australia

(Received 31 May 2006; accepted 18 September 2006; published 30 November 2006)

\begin{abstract}
A high brightness plasma ion source has been developed to address focused ion beam (FIB) applications not satisfied by the liquid metal ion source (LMIS) based FIB. The plasma FIB described here is capable of satisfying applications requiring high mill rates $\left(>100 \mu \mathrm{m}^{3} / \mathrm{s}\right)$ with non-gallium ions and has demonstrated imaging capabilities with sub- $100-\mathrm{nm}$ resolution. The virtual source size, angular intensity, mass spectra, and energy spread of the source have been determined with argon and xenon. This magnetically enhanced, inductively coupled plasma source has exhibited a reduced brightness $\left(\beta_{r}\right)$ of $5.4 \times 10^{3} \mathrm{~A} \mathrm{~m}^{-2} \mathrm{sr}^{-1} \mathrm{~V}^{-1}$, with a full width half maximum axial energy spread $(\Delta E)$ of $10 \mathrm{eV}$ when operated with argon. With xenon, $\beta_{r}=9.1$ $\times 10^{3} \mathrm{~A} \mathrm{~m}^{-2} \mathrm{sr}^{-1} \mathrm{~V}^{-1}$ and $\Delta E=7 \mathrm{eV}$. With these source parameters, an optical column with sufficient demagnification is capable of forming a sub-25-nm spot size at $30 \mathrm{keV}$ and $1 \mathrm{pA}$. The angular intensity of this source is nominally three orders of magnitude greater than a LMIS making the source more amenable to creating high current focused beams, in the regime where spherical aberration dominates the LMIS-FIB. The source has been operated on a two lens ion column and has demonstrated a current density that exceeds that of the LMIS-FIB for current greater than $50 \mathrm{nA}$. Source lifetime and current stability are excellent with inert and reactive gases. Additionally, it should be possible to improve both the brightness and energy spread of this source, such that the $\left(\beta_{r} / \Delta E^{2}\right)$ figure-of-merit could be within an order of magnitude of a LMIS. () 2006 American Vacuum Society.
\end{abstract}

[DOI: $10.1116 / 1.2366617]$

\section{INTRODUCTION}

For the past 20 years, state-of-the-art focused ion beam (FIB) systems have relied on the high brightness, moderate energy spread, ease-of-use, and robustness of the gallium liquid metal ion source (LMIS). These FIB systems typically deliver beam currents ranging from $1 \mathrm{pA}$ to $20 \mathrm{nA}$, focused into spot diameters of approximately $5-400 \mathrm{~nm}$ at $30-50 \mathrm{keV}$. As a point of reference, a $30 \mathrm{keV} / 20 \mathrm{nA}$ gallium beam sputters silicon (normal incidence) at a rate of $\sim 5.4 \mu \mathrm{m}^{3} / \mathrm{s}$. Hence, with a maximum beam current of $20 \mathrm{nA}$, mill volumes in excess of $20000 \mu \mathrm{m}^{3}$ require prohibitively long mill times (i.e., $>1 \mathrm{~h}$ ). In many instances there is a need to remove more than $1 \times 10^{6} \mu \mathrm{m}^{3}$ of material for large area cross-sectioning of integrated circuits microelectromechanical system (MEMS) failure analysis and prototyping, secondary-ion-mass spectroscopy (SIMS) and rapid transmission electron microscopy (TEM) sample preparation. The obvious solution is to increase the beam current. However, as seen in the LMIS curve of Fig. 1, the spot size increases rapidly (above $5 \mathrm{nA}$ ) with a concomitant drop in current density. This dependence can be understood, if one considers the optical properties of a generic two lens FIB column as a function of beam current or aperture angle. To a

\footnotetext{
${ }^{a)}$ Electronic mail: nsmith@feico.com
}

good approximation the focused beam diameter is determined by convolving the Gaussian image of the virtual source diameter $\left(d_{v}\right)$ with the first order chromatic and third order spherical aberration contributions from the FIB optics. At a specific linear magnification, a minimum of the summed contributions of these components occurs, according to Eq. (1). ${ }^{1}$

$$
d_{\mathrm{tot}}=\left(\left(d_{C_{s}}^{1.3}+d_{g}^{1.3}\right)^{2 / 1.3}+d_{C_{c}}^{2}\right)^{0.5},
$$

where $d_{\text {tot }}$ (beam width containing $50 \%$ of the beam current) is made up of contributions from spherical aberration $\left(d_{C_{s}}\right)$, chromatic aberration $\left(d_{C_{c}}\right)$, and the linear magnification of the virtual source $\left(d_{g}\right)$ (assuming axial energy spread and $d_{v}$ have Gaussian distributions), where

$$
\begin{aligned}
& d_{C_{s}}=0.5^{2.5} C_{s} \alpha_{i}^{3}, \\
& d_{C_{c}}=0.34 C_{c}\left(\frac{\Delta U_{\mathrm{FWHM}}}{U_{i}}\right) \alpha_{i}, \\
& d_{g}=d_{v} M,
\end{aligned}
$$

with the relationship between object and image side angles given by the Helmholtz-Lagrange relationship 


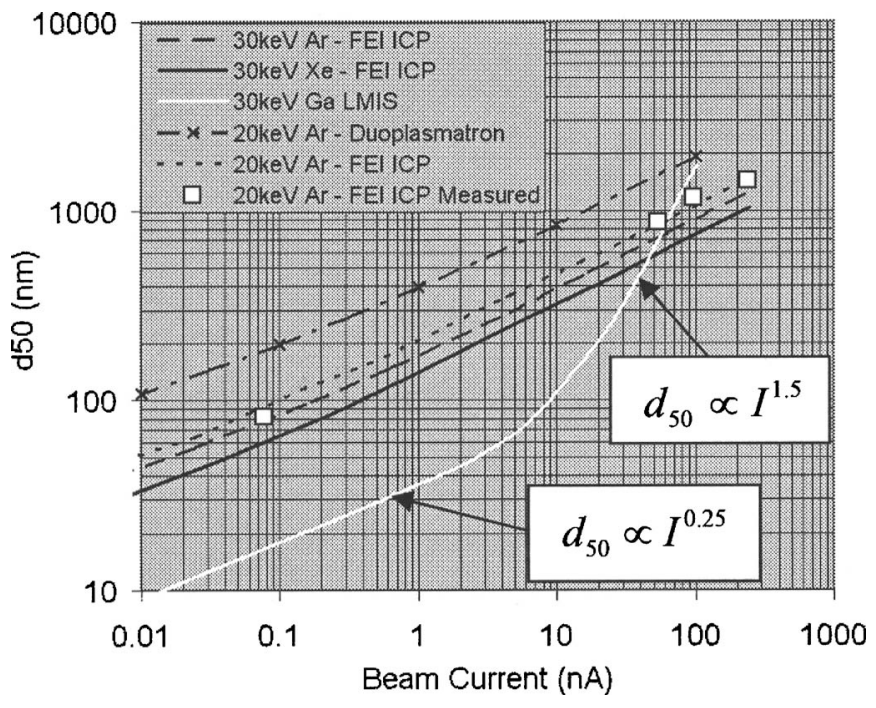

FIG. 1. Spot size data comparing state-of-the-art LMIS-FIB and Duoplasmatron with the FEI ICP source.

$$
M=\frac{\alpha_{o}}{\alpha_{i}} \sqrt{\frac{U_{o}}{U_{i}}},
$$

where $\alpha_{o}$ and $\alpha_{i}$ are the object and image side half-angles, $C_{s}$ and $C_{c}$ are the spherical and chromatic aberration coefficients, $U_{o}$ and $U_{i}$ are the object and image side beam energies, $\Delta U_{\mathrm{FWHM}}$ is the FWHM of the axial energy spread, and $M$ is the linear magnification.

Above $20 \mathrm{nA}$ the optical performance is dominated by spherical aberration. Increasing beam current necessitates an increase in $\alpha_{o}$ and an increased $M$ in order to attain the smallest spot size. $M$ increases proportionally to $\alpha_{o}^{3 / 2}$ (or $I^{3 / 4}$ ) if $C_{s}$ remains constant.

Typically, FIB probe forming optics operate with $M \ll 1$ in order to demagnify the virtual source size and the aberrations of only the second lens (objective) need to be considered. For a fixed working distance, the focal length of the objective lens changes only slightly across the full operating range of the FIB (i.e., $1 \mathrm{pA}-30 \mathrm{nA}$ ) and hence $C_{s}$ is nearly invariant. However, for $M \geqslant 1$ (optimum for $>1 \mathrm{nA}$ ), the aberration contribution from the condenser lens needs to be considered and, in fact, dominates $C_{s}$ [Eq. (6)] and the optimum spot size that can be attained. ${ }^{2}$

$$
C_{s}=C_{\mathrm{si}}+C_{\mathrm{so}} M^{4}\left(U_{i} / U_{o}\right)^{3 / 2},
$$

where $C_{s}$ is the total spherical aberration coefficient for the column referred to the image side, $C_{\mathrm{si}}$ is the image side spherical aberration coefficient for lens 2 only, and $C_{\mathrm{so}}$ is the object side spherical aberration coefficient for lens 1 only. The net effect is that in this high current regime, $M$ can no longer be increased to arrest spherical blur and $d_{s}$ increases proportionally to $I^{3 / 2}$, with $d_{\mathrm{tot}}=d_{s}$ (see LMIS curve in Fig. 1).

Another way of understanding this effect is to consider the image side brightness $\left(\beta_{i}\right)$ as a function of beam current $(I)$, where the reduced source brightness $\left(\beta_{\mathrm{rs}}\right)$ is taken to be $1 \times 10^{6} \mathrm{~A} \mathrm{~m}^{-2} \mathrm{sr}^{-1} \mathrm{~V}^{-1}$. Below $1 \mathrm{nA}$, the reduced image side brightness $\left(\beta_{\mathrm{ri}}\right)$ is $\sim 50 \%$ of the source brightness. This represents a final spot that is $\sqrt{2}$ larger than the Gaussian spot with an optimal balance of chromatic and Gaussian contributions. However, above $1 \mathrm{nA}$ the image side brightness drops precipitously, such that at $100 \mathrm{nA}, \quad \beta_{\mathrm{ri}}$ $\sim 330 \mathrm{~A} \mathrm{~m}^{-2} \mathrm{sr}^{-1} \mathrm{~V}^{-1}$. So, with this same optical configuration, $100 \mathrm{nA}$ could be focused into the same $1.5 \mu \mathrm{m}$ spot size, with $\beta_{\mathrm{rs}} \sim 660 \mathrm{~A} \mathrm{~m}^{-2} \mathrm{sr}^{-1} \mathrm{~V}^{-1}$ provided that the optical column can operate with $M \ll 1$ (i.e., $d_{v}>1 \mu \mathrm{m}$ ).

These conditions are approximately satisfied by a few plasma ion sources. For at least 40 years, the Duoplasmatron ion source has been the brightest commercially available plasma source ${ }^{3}$ for FIB/microprobe applications, with $\beta_{\mathrm{rs}}$ $=1130 \mathrm{~A} \mathrm{~m}^{-2} \mathrm{sr}^{-1} \mathrm{~V}^{-1}$. The main disadvantages of this source are the short lifetime and the ability to operate with only a limited number of gas/ion species. Recently Scipioni et $a l .{ }^{4}$ reported the source properties and FIB performance of a multicusp plasma source. In this case, $\beta_{\mathrm{rs}}$ is lower than the Duoplasmatron (550 A m $\mathrm{Ar}^{-2} \mathrm{~V}^{-1}$ ), but the source has significantly lower energy spread (i.e., $1-2 \mathrm{eV}$ vs $10-15 \mathrm{eV}$ for the Duoplasmatron), resulting in a much better $\left(\beta_{r} / \Delta E^{2}\right)$ figure-of-merit in chromatically limited regimes. The main disadvantages of this source are the internal cathodic element (limiting life and the number of available gas/ion species) and the marginal brightness for high current applications. Many other groups have also reported advances in plasma ion source brightness, ${ }^{5-7}$ anticipating unprecedented small spot sizes from theoretical spot size calculations.

This article describes the performance of a magnetically enhanced inductively coupled plasma (ICP) ion source that has been shown to meet the brightness and angular intensity requirements for high current inert-ion FIB operation. The ICP source has moderate axial energy spread, excellent beam purity and lifetime, as well as the ability to operate with a variety of inert and reactive gases. Initial FIB performance, using a standard two lens column, is reported here.

\section{SOURCE OVERVIEW AND EXPERIMENTAL SETUP}

The requirements for a commercially viable plasma ion source are high brightness, low energy spread, long/ predictable life, good beam current stability, high beam purity and the ability to select from a range of ion types (inert, reactive or molecular). DC plasma sources have a limited life due to cathode erosion (typically $250-500 \mathrm{~h}$ ), with lifetime scaling inversely with plasma density and hence source brightness. The ICP source described here has an external RF $(13.56 \mathrm{MHz})$ antenna that couples energy to the plasma electrons almost entirely inductively ${ }^{8}$ (therefore, there is no internal cathode erosion). Care has been taken to minimize capacitive coupling effects, as these can limit source lifetime and cause a broadened/bimodal axial energy spread in RF ion sources. ${ }^{9}$ The source has an automated gas delivery system which regulates source pressure (10-100 mTorr) and is made from materials compatible with UHV and high tem- 
perature operation. The source ions are extracted through a $200 \mu \mathrm{m}$ diameter aperture in the source electrode/anode, via a three-electrode extraction optical element.

Virtual source location and size, angular intensity, and brightness have been derived from FIB spot size measurements under specific operating conditions. Current density distributions in the focused spots have been determined to be nominally Gaussian, so that $12 \%-88 \%$ edge resolution measurements from secondary electron images are used to measure the $d_{50}$ spot size. One can determine angular intensity and virtual source size from beam current measurements and spot size data, with knowledge of the optical properties of the two lens FIB column and the virtual source location. The axial location of the virtual source has been experimentally determined [Eq. (7)] by measuring the spot size when operating the column with lens 1 only and then with lens 2 only $\left(d_{i 1}\right.$ and $d_{i 2}$ are the respective diameters of the Gaussian contribution to the spot), $u$ is the distance from the virtual source to the principle plane of lens $2, x$ is the distance between the principle planes of the two lenses, and $v$ is the distance from the second lens principle plane to the image. The optical properties of the lenses have been determined using Munro's optics program. ${ }^{10}$

$$
u=x\left(1-\left(\frac{d_{i 1}(v+x)}{d_{i 2} v}\right)\right)^{-1} .
$$

These experimentally determined source parameters have been used to predict the optimum spot size versus current for a broader range of operating conditions, with a subset of these proven experimentally. Milling experiments have been performed on a standard FEI tool (AT600).

\section{RESULTS}

\section{A. Mass spectral composition}

Beam purity is extremely important for many ion beam milling applications. Of course, the ion beam can always be mass filtered to improve the beam purity but this reduces the effective brightness of the source. One of the advantages of this ICP source is that there is negligible cathode sputter erosion. Provided that the plasma potential stays below $\sim 30 \mathrm{~V}$ with respect to the surrounding boundary, ions leave the plasma and do not sputter the material that encloses the plasma. No sputter erosion means no obvious end-of-life mechanism in the source and no sputtered contaminants in the beam that originate from source materials. After $\sim 800 \mathrm{~h}$ of operation the plasma source chamber shows no signs of sputter erosion.

In addition to minimizing sputter erosion of source materials, it is also important to have limited out-gassing into the plasma volume. With a gas throughput of $\sim 1$ $\times 10^{-4}$ Torr $1 / \mathrm{s}$, the outgas rate in the source needs to be $\leqslant 10^{-5}$ Torr $1 / \mathrm{s}$ to ensure that the beam composition is $\geqslant 90 \%$ of the chosen gas species.

Figure 2 shows a typical mass spectra when operating the source with xenon. Clearly, $99.0 \%$ of the ions in the mass

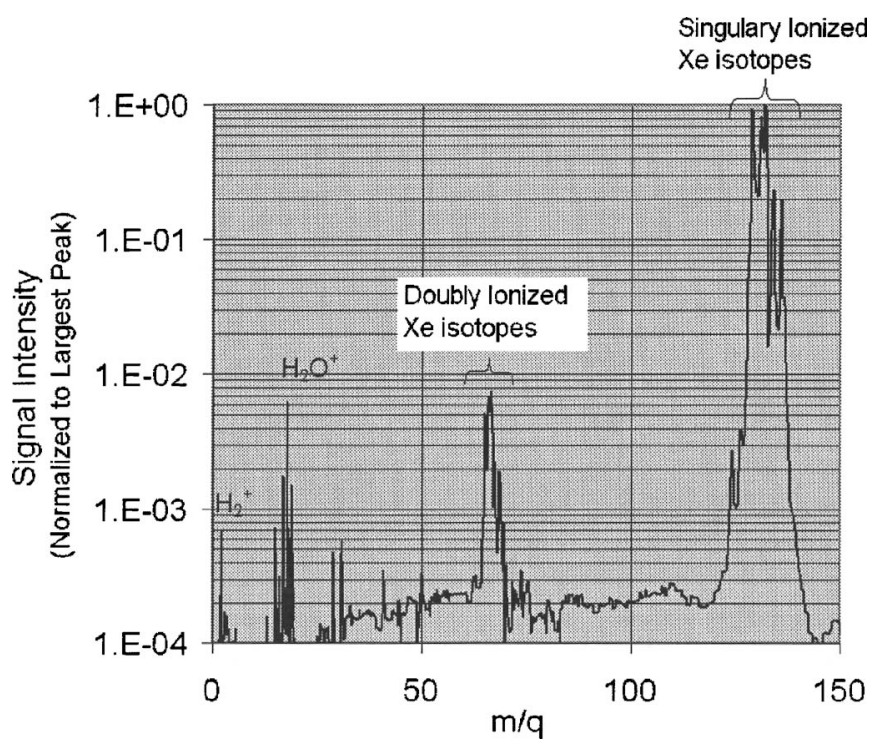

FIG. 2. Mass spectra for $300 \mathrm{~W}$ xenon plasma.

spectrum are $\mathrm{Xe}^{+}$isotopes, $0.75 \%$ are the doubly charged xenon ions, with $\sim 0.25 \%$ being impurity species below 20 amu.

\section{B. Axial energy distribution}

With a time-independent plasma potential, the axial energy spread of an ICP source is fundamentally limited by the "presheath" potential gradient. ${ }^{11}$ In order to reduce electron displacement currents in the plasma (which cause sympathetic modulations of the plasma potential) capacitive coupling of the time dependent voltage across the inductive antenna to the plasma must be minimized. A significant RF modulation of the plasma potential will result in a broad bimodal energy distribution and short source lifetime.

A magnetic sector mass spectrometer has been used to analyze the axial energy spread and to obtain mass spectra of the beam in order to optimize the source configuration. The energy resolution of the spectrometer is $<1.6 \mathrm{eV}$. Figure 3 shows the ion energy distribution of this new source operating with xenon and a RF power of $300 \mathrm{~W}$. The energy spread is quasisymmetric but does not fit well to a Gaussian distribution. Here $\Delta U_{\mathrm{FWHM}}=6.7 \mathrm{eV}$, while $\Delta U_{\mathrm{FW} 50}$ (energy width containing half the current) is $3.1 \mathrm{eV}$. For subsequent optics calculations a prefactor of 0.6 and a $\Delta U_{\mathrm{FW} 50}$ energy spread have been used in Eq. (3), instead of the respective values of a 0.34 prefactor and $\Delta U_{\mathrm{FWHM}}$, according to the Barth and Nykerk ${ }^{12}$ method.

Although the energy distribution is a single peak (not bimodal), it may still be broadened by a few $\mathrm{eV}$ due to a small time dependent modulation of the plasma potential. The proposed fundamental limit to the energy spread (solely due to the presheath potential gradient) is typically quoted as $\left(T_{e} / 2\right)$, where $T_{e}$ is the electron temperature in $\mathrm{eV}^{11}$ Since the electron temperature is $3-4 \mathrm{eV}$ (for this type of plasma) 


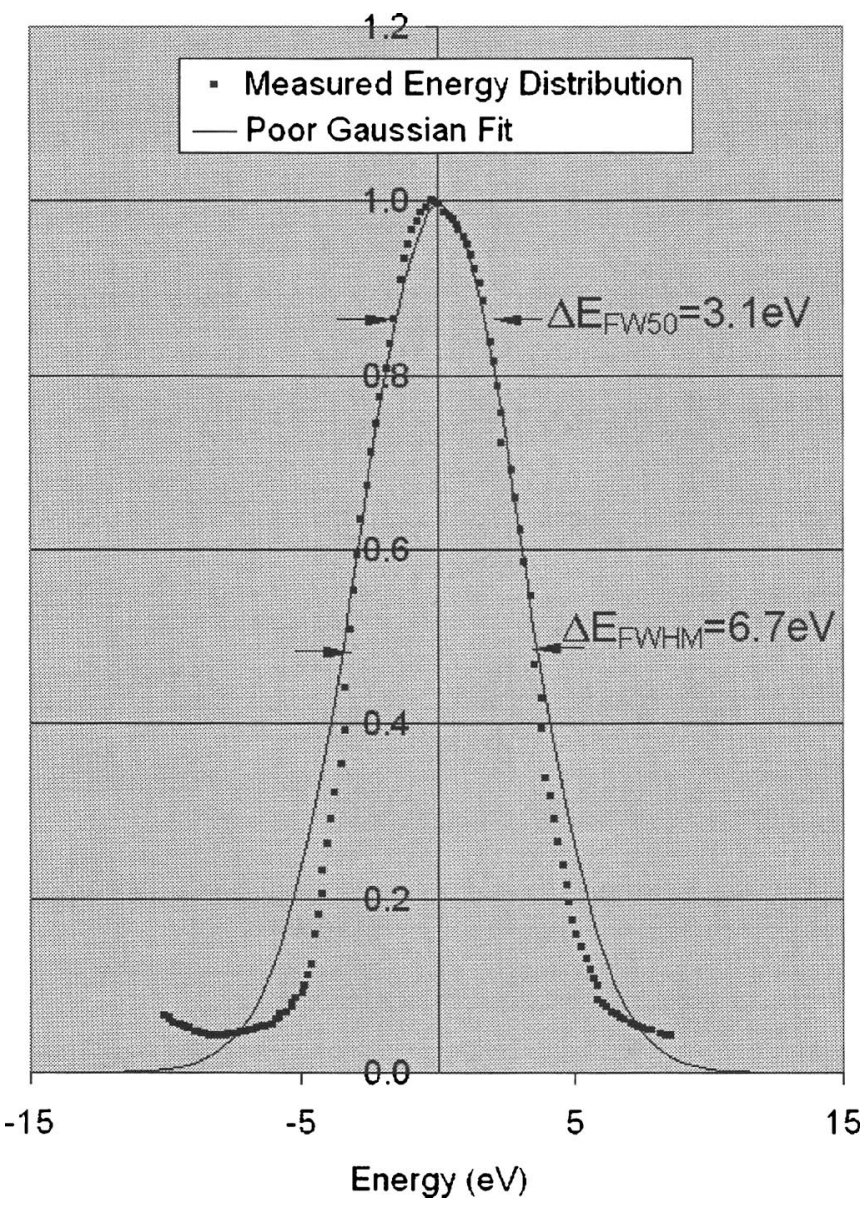

FIG. 3. Energy distribution for $300 \mathrm{~W}$ xenon plasma.

an axial energy spread of $1.5-2 \mathrm{eV}$ should be possible in the absence of the Boersch effect, charge exchange collisions, and plasma potential modulations.

\section{Source brightness and emittance}

Source brightness was determined by measuring spot size data from the plasma FIB and back-calculating the brightness, "looking back" from the first lens towards the threeelectrode extraction optics. This yields the "effective brightness," resulting from the current density at the source aperture, the thermal ion energy of plasma ions (determining the fundamental limit to the beam emittance), any particle interactions (i.e., Coulomb, ion scattering, charge-exchange interactions, etc.), nonlinear space charge effects, and aberrations from the extraction field. The effective brightness results from the convolution of all these emittance expanding effects and represents the source properties, as seen by the subsequent optical column. Brightness and emittance have the well known reciprocal relationship [Eq. (8)], where $\varepsilon$ is the transverse beam emittance ( $\mathrm{cm} \mathrm{rad}$ ), that can be determined at any plane that is transverse to the optical axis.

$$
\beta=I / \varepsilon^{2} \text {. }
$$

The axial location of the virtual source, its $d_{50}$ diameter, and the angular intensity of the beam have been determined for specific conditions (i.e., extraction field, plasma density, and plasma species). Variations in these parameters alter the shape of the beam's emittance beyond the extraction optics and hence the virtual source parameters (i.e., angular divergence might be exchanged for virtual source size, without necessarily affecting the emittance or brightness values). Although beam emittance has not been measured for a range of extraction conditions, we have one data point that indicates that there is some flexibility in beam emittance shape without significantly compromising the emittance area.

For a $300 \mathrm{~W}$ argon plasma and the three extraction electrodes biased with an $8.5 \mathrm{kV}$ extraction voltage and a final beam energy of $20 \mathrm{keV}$, the virtual source appears $\sim 13 \mathrm{~mm}$ in front of the plasma emissive surface with $d_{v}=15.3 \mu \mathrm{m}$ and the angular intensity is $I^{\prime}=19.8 \mathrm{~mA} / \mathrm{sr}$ so that the effective reduced brightness is $5400 \mathrm{~A} \mathrm{~m}^{-2} \mathrm{sr}^{-1} \mathrm{~V}^{-1}$. Altering the field distribution in the extraction optics ( $4 \mathrm{kV}$ extract potential and $20 \mathrm{keV}$ final energy) results in an apparently linear transformation of the beam's phase space (i.e., reshaped emittance, but with approximately the same value of emittance area) entering the first lens. In the latter case, the beam is more divergent $\left(I^{\prime}=8.1 \mathrm{~mA} / \mathrm{sr}\right)$ but the virtual source size is reduced $\left(d_{v}=9.5 \mu \mathrm{m}, \sim 2 \mathrm{~mm}\right.$ behind the plasma surface) such that $B_{r s}$ is nominally the same as with the initial field distribution.

Xenon has a lower first ionization potential than argon and a larger ionization cross-section resulting in a higher plasma density per unit of RF power. Higher plasma density results in a higher ion current density, such that the $300 \mathrm{~W}$ xenon plasma generates $60 \%$ more current density from the source aperture (i.e., $38 \mu \mathrm{A}$ through a $200 \mu \mathrm{m}$ aperture) than argon. With an extraction voltage of $11.5 \mathrm{kV}$ and a final beam energy of $20 \mathrm{keV}$, the virtual source appears $\sim 1 \mathrm{~mm}$ behind the plasma surface with $d_{v}=7.2 \mu \mathrm{m}, I^{\prime}=7.4 \mathrm{~mA} / \mathrm{sr}$, and the effective reduced brightness is $9100 \mathrm{~A} \mathrm{~m}^{-2} \mathrm{sr}^{-1} \mathrm{~V}^{-1}$.

These source parameters are good evidence that ion heating, stochastic Coulomb interactions, and optical aberrations in the extraction optics are very low. Even if one were to assume no emittance growth between the plasma surface and the entrance to the column optics (i.e., assume the measured brightness is at the thermal limit), the mean thermal ion energy would only be twice the ambient gas temperature (i.e., $\sim 0.05 \mathrm{eV}$ ) for both argon and xenon.

Xenon is the inert gas of choice for rapid milling applications due to its large sputter yield $(\sim 3.5$ times larger than argon at normal incidence in silicon), greater source brightness for a given RF power, and lower energy spread. However, much of the initial source data have been collected with argon as this is a more common choice. Initial $20 \mathrm{keV}$ argon data have been used to calculate the expected optimum spot size versus beam current and compared to the Duoplasmatron at the same energy (Fig. 1). These data were acquired at $20 \mathrm{keV}$, as this was a limitation at the time of the experiment. Experimental spot size data are included in the graph and agree well with the theoretical calculations. It should be noted that the column has not been configured yet to operate optimally across a broad range of beam currents. The three 
high current experimental data points were determined from secondary electron images of graphite, while operating with an extraction potential of $8.5 \mathrm{kV}$ and post-acceleration to $20 \mathrm{keV}$, with the column optics in a collimated mode. The lower current value has been acquired with the extraction potential at $4 \mathrm{kV}$ and final energy of $20 \mathrm{keV}$ in order to demonstrate a sub-100-nm spot. The column demagnification is presently limited to $\sim 200 \times$, resulting in the reduced $d_{v}$ condition being better matched to low current beams. The energy spread under these conditions is marginally lower $(\sim 7 \mathrm{eV}$ vs $10 \mathrm{eV})$, explaining why the measured spot sits slightly off the calculated curve for $20 \mathrm{keV} \mathrm{Ar}^{+}$.

The solid and dashed lines in Fig. 1 show the calculated performance for this particular column operating with an optimal angular acceptance and linear magnification. Practically, this means using the appropriate angular defining aperture sizes and introducing a third lens for the ultimate low current/high resolution performance.

These generic FIB optics are able to operate optimally over a short range of high currents $(\sim 50-250 \mathrm{nA})$ with this plasma source without rapid erosion of the column beam defining aperture. However, at low currents the optimal column magnification puts a crossover very close to the aperture resulting in rapid erosion. With the source parameters sufficiently characterized, an optimized column can be designed to give long aperture life and a broad range of optimal operation that will match the curves in Fig. 1, from $1 \mathrm{pA}$ to several hundreds of nanoamperes.

\section{CONCLUSIONS}

The critical source parameters for a magnetically enhanced inductively coupled plasma ion source have been measured. The source has been operated on a two lens FIB column and has demonstrated sub-100-nm resolution with
$77 \mathrm{pA}$ of xenon at $20 \mathrm{keV}$. From these data, an extrapolation to a sub-25-nm, $1 \mathrm{pA}, 30 \mathrm{keV}$ xenon beam can be made. If the proposed limit to the axial energy spread can be reached $(2 \mathrm{eV})$, the current density in the low current chromatically limited regime will be only an order of magnitude lower than the LMIS. Above $50 \mathrm{nA}$, the energy spread has negligible influence and already this source exhibits higher current densities than the LMIS-FIB. In fact, it should be noted that since the LMIS-FIB beam is heavily dominated by spherical blur above $10 \mathrm{nA}$, the beam shape is non-Gaussian with significant beam tails. Hence, the full advantage of this plasma source is not fully reflected by the $d_{50}$ spot size and, in fact, the plasma source may well be the beam of choice for milling applications at currents in the 30-40 nA regime. With this new system (using beam currents up to $250 \mathrm{nA}$ and $1 \mu \mathrm{m}$ spot size) high current milling applications requiring $>1 \times 10^{6} \mu \mathrm{m}^{3}$ of material to be milled can routinely be performed at a speed that is $\sim 20$ times faster than the $20 \mathrm{nA}$ LMIS-FIB.

${ }^{1}$ J. E. Barth and P. Kruit, Optik (Stuttgart) 101, 101 (1996).

${ }^{2}$ J. Orloff, J. Vac. Sci. Technol. B 5, 175 (1987).

${ }^{3}$ C. D. Coathe and J. V. P. Long, Rev. Sci. Instrum. 66, 1018 (1995).

${ }^{4}$ L. Scipioni, D. Stewart, D. Ferranti, and A. Saxonis, J. Vac. Sci. Technol. B 18, 3194 (2000)

${ }^{5}$ X. Jiang, Q. Ji, A. Chang, and K. N. Leung, Rev. Sci. Instrum. 74, 2288 (2003).

${ }^{6}$ S. K. Guharay and J. Orloff, IEEE Trans. Plasma Sci. 33, 1911 (2005).

${ }^{7}$ S. N. Mordyk, V. I. Voznyy, I. Miroshnichenko, A. G. Ponomarev, V. E. Storizhko, and B. Sulkio-Cleff, Rev. Sci. Instrum. 75, 1922 (2004).

${ }^{8}$ J. Hopwood, Plasma Sources Sci. Technol. 1, 109 (1992).

${ }^{9}$ J. Hopwood, Appl. Phys. Lett. 62, 940 (1993).

${ }^{10} \mathrm{E}$. Munro, in Electron Optics and Computer Aided Design, edited by P. Hawkes (Academic, New York, 1973).

${ }^{11}$ K.-U. Riemann, J. Phys. D 24, 493 (1991).

${ }^{12}$ J. E. Barth and M. D. Nykerk, Nucl. Instrum. Methods Phys. Res. A 427, 86 (1999). 